\title{
EFFECTS ON THE DISTANCE LAPLACIAN SPECTRUM OF GRAPHS WITH CLUSTERS BY ADDING EDGES*
}

\author{
ROBERTO C. DÍAZ ${ }^{\dagger}$ AND OSCAR ROJO ${ }^{\dagger}$
}

\begin{abstract}
All graphs considered are simple and undirected. A cluster in a graph is a pair of vertex subsets $(C, S)$, where $C$ is a maximal set of cardinality $|C| \geq 2$ of independent vertices sharing the same set $S$ of $|S|$ neighbors. Let $G$ be a connected graph on $n$ vertices with a cluster $(C, S)$ and $H$ be a graph of order $|C|$. Let $G(H)$ be the connected graph obtained from $G$ and $H$ when the edges of $H$ are added to the edges of $G$ by identifying the vertices of $H$ with the vertices in $C$. It is proved that $G$ and $G(H)$ have in common $n-|C|+1$ distance Laplacian eigenvalues, and the matrix having these common eigenvalues is given, if $H$ is the complete graph on $|C|$ vertices then $\partial-|C|+2$ is a distance Laplacian eigenvalue of $G(H)$ with multiplicity $|C|-1$, where $\partial$ is the transmission in $G$ of the vertices in $C$. Furthermore, it is shown that if $G$ is a graph of diameter at least 3 , then the distance Laplacian spectral radii of $G$ and $G(H)$ are equal, and if $G$ is a graph of diameter 2, then conditions for the equality of these spectral radii are established. Finally, the results are extended to graphs with two or more disjoint clusters.
\end{abstract}

Key words. Cluster, Pendant vertices, Distance Laplacian matrix, Distance Laplacian eigenvalues, Distance spectral radius.

AMS subject classifications. 05E30, 15A18, 05C50.

1. Introduction. Let $G=(V(G), E(G))$ be a simple undirected graph on $n$ vertices with vertex set $V(G)$ and edge set $E(G)$. Let $D(G)$ be the diagonal matrix of order $n$ whose $(i, i)$-entry is the degree of the $i-t h$ vertex of $G$ and let $A(G)$ be the adjacency matrix of $G$. The matrices $L(G)=D(G)-A(G)$ and $Q(G)=D(G)+A(G)$ are the Laplacian and signless Laplacian matrices of $G$, respectively. It is known that $L(G)$ and $Q(G)$ are both positive semidefinite matrices. Thus, for each of these matrix, the corresponding spectral radius is an eigenvalue. They are called the Laplacian spectral radius and the signless Laplacian spectral radius of $\mathrm{G}$, respectively. It is immediate that $(0, \mathbf{1})$ is an eigenpair of $L(G)$, where $\mathbf{1}$ is the all ones vector. Fiedler [7] proved that $G$ is a connected graph if and only if the second smallest eigenvalue of $L(G)$ is positive. This eigenvalue is called the algebraic connectivity of $G$.

As usual, $K_{1, c}$ denotes a star on $c+1$ vertices, $K_{n}$ is a complete graph and $P_{n}$ is a path of $n$ vertices.

The distance between $u, v \in V(G)$ for a connected graph $G$, denoted by $d(u, v)$, is the length of the shortest path connecting $u$ and $v$. The Wiener index $W(G)$ of the graph $G$ is

$$
W(G)=\frac{1}{2} \sum_{u, v \in V(G)} d(u, v)
$$

and the transmission $\operatorname{Tr}(v)$ of a vertex $v \in V(G)$ is the sum of the distances from $v$ to all other vertices of

\footnotetext{
*Received by the editors on October 8, 2018. Accepted for publication on September 8, 2019. Handling Editor: Ravi Bapat. Corresponding Author: Oscar Rojo.

†Departamento de Matemáticas, Universidad Católica del Norte, Antofagasta, Casilla 1280, Chile (rdiaz01@ucn.cl, orojo@ucn.cl). The research of R. Díaz was supported by Conicyt-Fondecyt de Postdoctorado 2017, no. 3170065, Chile. The research of O. Rojo was supported by Project Fondecyt Regular 1170313, Chile.
} 
$G$, that is,

$$
\operatorname{Tr}(v)=\sum_{u \in V(G)} d(v, u)
$$

The graph $G$ is said to be $k$ - transmission regular if $\operatorname{Tr}(v)=k$ for each vertex $v \in V(G)$. The distance matrix $\mathcal{D}(G)=\left(d_{i, j}\right)$ of $G$ is an $n \times n$ matrix indexed by the vertices of $G$ in which $d_{i, j}=d\left(v_{i}, v_{j}\right)$. The eigenvalues of $\mathcal{D}(G)$ are called the distance eigenvalues of $G$ and they are denoted by

$$
\partial_{1}(G) \geq \partial_{2}(G) \geq \cdots \geq \partial_{n}(G)
$$

Clearly, $\mathcal{D}\left(K_{n}\right)=A\left(K_{n}\right)$ and then $\partial_{1}\left(K_{n}\right)=n-1$ and $\partial_{i}(G)=-1$ for $i=2, \ldots, n$.

In [2], Aouchiche and Hansen introduce the distance Laplacian matrix $\mathcal{L}(G)$ and the signless Laplacian matrix $\mathcal{Q}(G)$ as follows:

$$
\mathcal{L}(G)=\operatorname{Tr}(G)-\mathcal{D}(G)
$$

and

$$
\mathcal{Q}(G)=\operatorname{Tr}(G)+\mathcal{D}(G),
$$

where

$$
\operatorname{Tr}(G)=\operatorname{diag}\left[\operatorname{Tr}\left(v_{1}\right), \operatorname{Tr}\left(v_{2}\right), \ldots, \operatorname{Tr}\left(v_{n}\right)\right]
$$

is the diagonal matrix of the vertex transmissions in $G$.

The eigenvalues of $\mathcal{L}(G)$ and $\mathcal{Q}(G)$ are called the distance Laplacian eigenvalues and the distance signless Laplacian eigenvalues of $G$, and they are denoted by

$$
\partial_{1}^{L}(G) \geq \partial_{2}^{L}(G) \geq \cdots \geq \partial_{n}^{L}(G)
$$

and

$$
\partial_{1}^{Q}(G) \geq \partial_{2}^{Q}(G) \geq \cdots \geq \partial_{n}^{Q}(G),
$$

respectively.

From the above definitions, it follows that $\partial_{n}^{L}(G)=0$ with eigenvector 1 and

$$
\operatorname{trace}(\mathcal{L}(G))=\operatorname{trace}(\mathcal{Q}(G))=2 W(G) .
$$

Clearly, if $G$ is a $k$ - transmission regular graph, then

$$
\mathcal{L}(G)=k I_{n}-\mathcal{D}(G)
$$

and

$$
\mathcal{Q}(G)=k I_{n}+\mathcal{D}(G)
$$


where $I_{n}$ is the identity matrix of order $n$; and, for $i=1, \ldots, n$,

$$
\partial_{i}^{\mathcal{L}}(G)=k-\partial_{n-i+1}(G)
$$

and

$$
\partial_{i}^{\mathcal{Q}}(G)=k+\partial_{i}(G) .
$$

Definition 1.1. ([4, 9]) A cluster in a graph $G$ is a pair of vertex subsets $(C, S)$, where $C$ is a maximal set of independent vertices of cardinality $|C| \geq 2$ sharing the same set $S$ of $|S|$ neighbors.

Example 1. The star $K_{1,|C|}$ is a subgraph of a graph $G$ then $(C, S)$ is a cluster in $G$, where $C$ is the set of the pendant vertices and $S=\{v\}$, being $v$ the root vertex of the star.

EXAMPLE 2. Let $G$ :

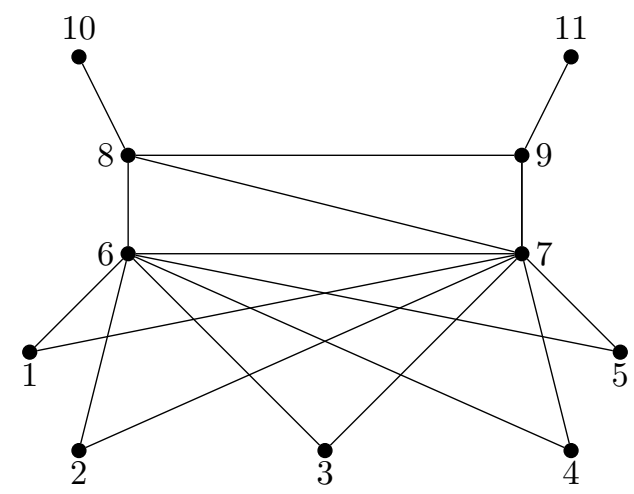

We see that $(C, S)$, where $C=\{1,2,3,4,5\}$ and $S=\{6,7\}$ is a cluster in $G$.

Definition 1.2. Let $G$ be a graph having a cluster $(C, S)$. Let $H$ be a graph of order $|C|$. Let $G(H)$ be the graph obtained from $G$ and $H$ when the edges of $H$ are added to the edges of $G$ by identifying the vertices of $H$ with the vertices in $C$.

From Definition 1.2, $G(H)$ is a graph with vertex set $V(G(H))=V(G)$ and edge set $E(G(H))=$ $E(G) \cup E(H)$.

Example 3. Let $G$ be the graph in Example 2, and let $H=P_{5}$. Then $G(H)$ is the graph displayed below:

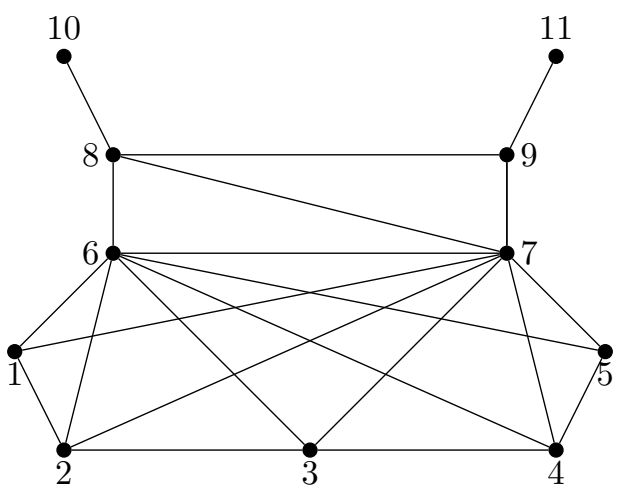


The Laplacian and signless Laplacian spectra of a graph $G$ with a cluster $(C, S)$ are studied in [1]. In [6] and [12], respectively, the authors prove that the Laplacian spectral radius and algebraic connectivity of a graph do not change when edges are added among the pendant vertices. The effects on some others spectral invariants are determined in [10] and [11].

Definition 1.3. Let $\left(C_{1}, S_{1}\right)$ and $\left(C_{2}, S_{2}\right)$ be clusters in a graph $G$. We say that $\left(C_{1}, S_{1}\right)$ and $\left(C_{2}, S_{2}\right)$ are disjoint if $C_{1} \cap C_{2}=\phi$ and $S_{1} \cap S_{2}=\phi$.

EXAMPLE 4. Let $G$ :

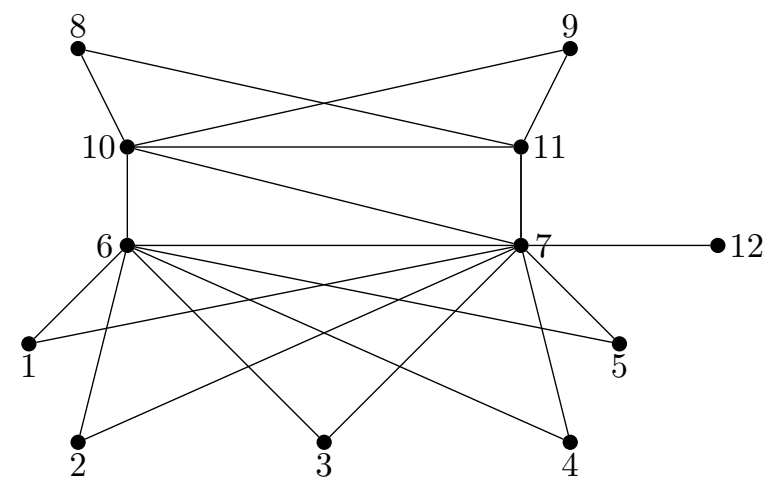

We see that $\left(C_{1}, S_{1}\right), C_{1}=\{1,2,3,4,5\}, S_{1}=\{6,7\}$ and $\left(C_{2}, S_{2}\right), C_{2}=\{8,9\}, S_{2}=\{10,11\}$, are disjoint clusters in $G$.

Definition 1.4. Let $N_{k}=\{1,2, \ldots, k\}$ and let $G$ be a graph having pairwise disjoint clusters $\left(C_{1}, S_{1}\right)$, $\ldots,\left(C_{k}, S_{k}\right)$. For $i \in N_{k}$, let $H_{i}$ be a graph of order $\left|C_{i}\right|$. Let $G\left(H_{i}: i \in N_{k}\right)$ be the graph obtained from $G$ and the graphs $H_{i}$ when the edges of $H_{i}$ are added to the edges of $G$ by identifying the vertices of $H_{i}$ with the vertices in $C_{i}$ for $i \in N_{k}$.

From this definition, we have

$$
V\left(G\left(H_{i}: i \in N_{k}\right)\right)=V(G)
$$

and

$$
E\left(G\left(H_{i}: i \in N_{k}\right)\right)=E(G) \cup E\left(H_{1}\right) \cup \cdots \cup E\left(H_{k}\right) .
$$

In [4], it is proved that the graphs $G$ and $G\left(H_{i}: i \in N_{k}\right)$ have $n-\sum_{j=1}^{k}\left|C_{j}\right|+k$ Laplacian eigenvalues in common, among them the Laplacian spectral radii and algebraic connectivities.

The zero matrix of the appropriate order is denoted by 0 . Furthermore, $I_{m}$ is the identity matrix of order $m, \mathbf{1}_{c}$ is the column vector of ones of size $c, A^{T}$ is the transpose of $A$ and $C_{0} l_{i}(A)$ denotes the $i-t h$ column of $A$.

From now on, we assume that $G$ is a connected graph, $G(H)$ and $G\left(H_{i}: i \in N_{k}\right)$ are as in Definition 1.2 and Definition 1.4, respectively. Thus, $G(H)$ and $G\left(H_{i}: i \in N_{k}\right)$ are connected graphs.

In this paper, we prove that $G$ and $G(H)$ have in common $n-|C|+1$ distance Laplacian eigenvalues, and we determine the matrix having these common eigenvalues; if $H=K_{|C|}$, then $\partial-|C|+2$ is a distance 
Laplacian eigenvalue of $G\left(K_{|C|}\right)$ with multiplicity $|C|-1$, where $\partial$ is the transmission in $G$ of the vertices in $C$. Furthermore, we show that if $G$ is a graph of diameter at least 3, then the distance Laplacian spectral radii of $G$ and $G(H)$ are equal, and if $G$ is a graph of diameter 2, then we derive the conditions for the equality of these spectral radii. Finally, we extend the results to graphs with two or more disjoint clusters.

2. Preliminaries. In this section, we present some preliminaries results that will play important roles in our work.

The next two theorems are due to Aouchiche and Hansen and we state them using the notation introduced above.

TheOREm 2.1. ([3]) Let $G$ be a connected graph on $n$ vertices with a cluster $(C, S)$. Then,

1. the vertices in $C$ have the same transmission, and

2. $\partial+2$ is an eigenvalue of $\mathcal{L}(G)$ with multiplicity at least $|C|-1$, where $\partial$ is the transmission in $G$ of the vertices in $C$.

TheOREM 2.2. ([3]) Let $G$ be a connected graph on $n$ vertices with a cluster $(C, S)$. Consider the graph $G\left(K_{|C|}\right)$ as in Definition 2. Then,

1. in the graph $G\left(K_{|C|}\right)$, the vertices in $C$ have the same transmission, and

2. $\partial+1$ is an eigenvalue of $\mathcal{L}\left(G\left(K_{|C|}\right)\right)$ with multiplicity at least $|C|-1$, where $\partial$ is the transmission in $G\left(K_{|C|}\right)$ of the vertices in $C$.

COROLlary 2.3. Let $G$ be a connected graph of order $n$ with a cluster $(C, S)$. Let $\partial$ be the transmission in $G$ of the vertices in $C$. Then the transmission in $G\left(K_{|C|}\right)$ of the vertices in $C$ is $\partial-|C|+1$.

Proof. Let $\varphi$ be the transmission in $G\left(K_{|C|}\right)$ of the vertices in $C$. Then, for any $u \in C$, we have

$$
\partial=2(|C|-1)+|S|+\sum_{v \in V(G) \backslash(C \cup S)} d(u, v),
$$

and

$$
\varphi=(|C|-1)+|S|+\sum_{v \in V(G) \backslash(C \cup S)} d(u, v) .
$$

Therefore, $\varphi=\partial-|C|+1$.

COROLlary 2.4. Let $G$ be a connected graph of order $n$ with a cluster $(C, S)$. Let $\partial$ be the transmission in $G$ of the vertices in $C$. Then, $\partial-|C|+2$ is a distance Laplacian eigenvalue of $G\left(K_{|C|}\right)$ with multiplicity at least $|C|-1$.

Proof. The proof follows from Theorem 2.2 and Corollary 2.3.

TheOREm 2.5. ([13]) Let $G \neq K_{n}$ be a connected graph with maximum transmission $D_{1}$. Then

$$
\partial_{1}^{L}(G) \geq D_{1}+2 .
$$

Moreover, if $\operatorname{diam}(G) \geq 3$, then $\partial_{1}^{L}(G)>D_{1}+2$.

TheOREM 2.6. ([2]) Let $G$ be a connected graph on $n$ vertices and $m \geq n$ edges. Consider the connected graph $\widetilde{G}$ obtained from $G$ by the deletion of an edge. If $\left(\partial_{1}^{L}, \partial_{2}^{L}, \ldots, \partial_{n}^{L}\right)$ and $\left(\tilde{\partial}_{1}^{L}, \tilde{\partial}_{2}^{L}, \ldots, \tilde{\partial}_{n}^{L}\right)$ denote the distance Laplacian spectra of $G$ and $\widetilde{G}$, respectively, then $\tilde{\partial}_{i}^{L} \geq \partial_{i}^{L}$ for all $i=1, \ldots, n$. 
We recall that a circulant matrix is any square matrix in which each row is rotated one element to the right relative to the preceding row. More precisely, a matrix of the form

$$
C=\left[\begin{array}{ccccc}
c_{0} & c_{1} & \cdots & c_{n-2} & c_{n-1} \\
c_{n-1} & c_{0} & c_{1} & & c_{n-2} \\
\vdots & c_{n-1} & c_{0} & \ddots & \vdots \\
c_{2} & & \ddots & \ddots & c_{1} \\
c_{1} & c_{2} & \cdots & c_{n-1} & c_{0}
\end{array}\right]
$$

is a circulant matrix of order $n \times n$.

A general and remarkable result on circulant matrices is:

THEOREM 2.7. ([5]) The normalized eigenvectors of any circulant matrix of order $n \times n$ are

$$
\boldsymbol{x}_{j}=\frac{1}{\sqrt{n}}\left[1, \omega_{j}, \omega_{j}{ }^{2}, \ldots, \omega_{j}^{n-1}\right]^{T},
$$

$j=0,1, \ldots, n-1$, where $\omega_{j}=\exp \frac{2 i j \pi}{n}$ and $i=\sqrt{-1}$.

An immediate consequence of Theorem 2.7 is:

COROllary 2.8. Circulant matrices of the same order commute.

3. Main results. The graphs $G$ and $G(H)$ have the same set of vertices. We label the vertices of $G$ as follows: The labels $1,2, \ldots,|C|$ are for the vertices of $C$, the labels $|C|+1,|C|+2, \ldots,|C|+|S|$ are for the vertices in $S$ and the labels $|C|+|S|+1,|C|+|S|+2, \ldots, n$ are for the remaining vertices of $G$. This labeling is illustrated in Example 2. In particular, for the graphs $G$ and $G\left(K_{|C|}\right)$, we obtain

$$
\mathcal{L}(G)=\left[\begin{array}{cc}
E & X \\
X^{T} & Y
\end{array}\right]
$$

with

$$
E=\left[\begin{array}{cccc}
\partial & -2 & \cdots & -2 \\
-2 & \partial & \ddots & \vdots \\
\vdots & \ddots & \ddots & -2 \\
-2 & \cdots & -2 & \partial
\end{array}\right]
$$

and

$$
\mathcal{L}\left(G\left(K_{|C|}\right)\right)=\left[\begin{array}{cc}
F & X \\
X^{T} & Y
\end{array}\right]
$$

with

$$
F=\left[\begin{array}{cccc}
\partial-|C|+1 & -1 & \cdots & -1 \\
-1 & \partial-|C|+1 & \ddots & \vdots \\
\vdots & \ddots & \ddots & -1 \\
-1 & \cdots & -1 & \partial-|C|+1
\end{array}\right]
$$


where the circulant matrices $E$ and $F$ are both of order $|C| \times|C|$, the entries of $X$ are the negatives of the distances of the vertices in $C$ to the vertices $|C|+1, \ldots, n$, the diagonal entries of $Y$ are the transmissions of the vertices $|C|+1, \ldots, n$ and its off-diagonal entries are the negatives of the distances between these vertices.

TheOREM 3.1. Let $G$ be a connected graph having a cluster $(C, S)$. Let $\partial$ be the transmission in $G$ of the vertices in $C$. Then the matrices $\mathcal{L}(G)$ and $\mathcal{L}\left(G\left(K_{|C|}\right)\right)$ share $n-|C|+1$ eigenvalues and these are the eigenvalues of the $(n-|C|+1) \times(n-|C|+1)$ singular matrix

$$
T=\left[\begin{array}{cc}
\partial-2|C|+2 & \boldsymbol{y}^{T} \\
\boldsymbol{y} & Y
\end{array}\right]
$$

where

$$
\boldsymbol{y}=\left[\begin{array}{llll}
-\sqrt{|C|} d_{1,|C|+1} & -\sqrt{|C|} d_{1,|C|+2} & \cdots & -\sqrt{|C|} d_{1, n}
\end{array}\right]^{T} .
$$

Proof. We have

$$
E=2 \mathcal{L}\left(K_{|C|}\right)+(\partial-2|C|+2) I_{|C|}
$$

Then the eigenvalues of $E$ are $\partial+2$ with multiplicity $|C|-1$ and the single eigenvalue $\partial-2|C|+2$. For the matrix $\mathrm{F}$, we have

$$
F=\mathcal{L}\left(K_{|C|}\right)+(\partial-2|C|+2) I_{|C|} .
$$

Then the eigenvalues of $F$ are $\partial-|C|+2$ with multiplicity $|C|-1$ and the single eigenvalue $\partial-2|C|+2$. Theorem 2.7 allows us to construct an orthogonal matrix

$$
U=\left[\mathbf{x}_{1}, \ldots, \mathbf{x}_{|C|-1}, \frac{1}{\sqrt{|C|}} \mathbf{1}_{|C|}\right]
$$

whose columns are eigenvectors of the real circulant matrices $E$ and $F$. We observe that

$$
U^{T} X=\left[\begin{array}{cccc}
0 & 0 & \cdots & 0 \\
\vdots & \vdots & & \vdots \\
0 & 0 & \cdots & 0 \\
y_{1} & y_{2} & \cdots & y_{n-|C|}
\end{array}\right]
$$

where $y_{i}=-\sqrt{|C|} d_{1,|C|+i}$ for $i=1, \ldots, n-|C|$.

Let

$$
Q=\left[\begin{array}{cc}
U & 0 \\
0 & I_{n-|C|}
\end{array}\right]
$$

Clearly, $Q$ is an $n \times n$ orthogonal matrix. Hence, 


$$
\begin{aligned}
& Q^{T} \mathcal{L}(G) Q=\left[\begin{array}{cc}
U^{T} & 0 \\
0 & I_{n-|C|}
\end{array}\right]\left[\begin{array}{cc}
E & X \\
X^{T} & Y
\end{array}\right]\left[\begin{array}{cc}
U & 0 \\
0 & I_{n-|C|}
\end{array}\right]=\left[\begin{array}{cc}
U^{T} E U & U^{T} X \\
X^{T} U & Y
\end{array}\right] \\
& =\left[\begin{array}{c}
{\left[\begin{array}{cccc}
\partial+2 & 0 & \cdots & 0 \\
0 & \ddots & \ddots & \vdots \\
\vdots & \ddots & \partial+2 & 0 \\
0 & \cdots & 0 & \partial-2|C|+2
\end{array}\right]} \\
{\left[\begin{array}{cccc}
0 & \cdots & 0 & y_{1} \\
\vdots & & \vdots & y_{2} \\
0 & \cdots & 0 & \vdots \\
0 & \cdots & 0 & y_{n-|C|}
\end{array}\right]}
\end{array}\right] \\
& =\left[\begin{array}{ccc}
(\partial+2) I_{|C|-1} & 0 & 0 \\
0 & \partial-2|C|+2 & \mathbf{y}^{T} \\
0 & y & Y
\end{array}\right]=\left[\begin{array}{cc}
(\partial+2) I_{|C|-1} & 0 \\
0 & T
\end{array}\right] \text {. }
\end{aligned}
$$

Since $Q^{T} \mathcal{L}(G) Q$ and $\mathcal{L}(G)$ are similar matrices, it follows that the distance Laplace eigenvalues of $G$ are $\partial+2$ with multiplicity $|C|-1$ and the eigenvalues of the matrix $T$. Similarly,

$$
\begin{aligned}
& Q^{T} \mathcal{L}\left(G\left(K_{|C|}\right)\right) Q=\left[\begin{array}{cc}
U^{T} & 0 \\
0 & I_{n-|C|}
\end{array}\right]\left[\begin{array}{cc}
F & X \\
X^{T} & Y
\end{array}\right]\left[\begin{array}{cc}
U & 0 \\
0 & I_{n-|C|}
\end{array}\right] \\
& =\left[\begin{array}{cccc}
\partial-|C|+2 & 0 & \cdots & 0 \\
0 & \ddots & \ddots & \vdots \\
\vdots & \ddots & \partial-|C|+2 & 0 \\
0 & \cdots & 0 & \partial-2|C|+2
\end{array}\right]\left[\begin{array}{cccc}
0 & 0 & \cdots & 0 \\
\vdots & \vdots & & \vdots \\
0 & 0 & \cdots & 0 \\
y_{1} & y_{2} & \cdots & y_{n-|C|}
\end{array}\right] \\
& =\left[\begin{array}{ccc}
(\partial-|C|+2) I_{|C|-1} & 0 & 0 \\
0 & \partial-2|C|+2 & \mathbf{y}^{T} \\
0 & y & Y
\end{array}\right]=\left[\begin{array}{cc}
(\partial-|C|+2) I_{|C|-1} & 0 \\
0 & T
\end{array}\right] \text {. }
\end{aligned}
$$

The matrices $Q^{T} \mathcal{L}\left(G\left(K_{|C|}\right)\right) Q$ and $\mathcal{L}\left(G\left(K_{|C|}\right)\right)$ are similar. Then the distance Laplacian eigenvalues of $G\left(K_{|C|}\right)$ are $\partial-|C|+2$ with multiplicity $|C|-1$ and the eigenvalues of $T$. Observe that $T$ is a singular matrix. In addition, since $T$ is an $(n-|C|+1) \times(n-|C|+1)$ matrix whose eigenvalues are eigenvalues of $\mathcal{L}(G)$ and $\mathcal{L}\left(G\left(K_{|C|}\right)\right)$, the proof is complete. 
THEOREM 3.2. Let $G$ be a connected graph on $n$ vertices with a cluster $(C, S)$. Let $H$ be a graph of order $|C|$. Then $\mathcal{L}(G)$ and $\mathcal{L}(G(H))$ have in common the eigenvalues of the matrix $T$, given in (3.1), of order $(n-|C|+1) \times(n-|C|+1)$.

Proof. Applying Theorem 2.6, we get

$$
\partial_{i}^{L}\left(G\left(K_{|C|}\right)\right) \leq \partial_{i}^{L}(G(H)) \leq \partial_{i}^{L}(G)
$$

for $i=1, \ldots, n$. From Theorem 3.1, $\mathcal{L}(G)$ and $\mathcal{L}\left(G\left(K_{|C|}\right)\right)$ have in common the $n-|C|+1$ eigenvalues of the matrix $T$ in (3.1). Thus, using this fact in (3.2), the result follows.

LEMMA 3.3. Let $G$ be a connected graph of order $n$ with a cluster $(C, S)$ such that $|C|+|S|<n$ and $\operatorname{diam}(G)=2$. Let $\partial$ be the transmission in $G$ of the vertices in $C$. If $\partial_{1}^{L}(G)=\partial+2$, then each vertex in $S$ is connected to every vertex in $R=V(G) \backslash(C \cup S)$.

Proof. To obtain a contradiction, suppose that the assertion of the lemma is false. By Theorem 2.6, we may assume without loss of generality that each vertex in $S$ is connected to every vertex in $R$ except for the vertices $|C|+|S|$ in $S$ and $n$ in $R$. Let $\widetilde{G}$ the graph obtained from $G$ adding the edge connecting the vertex $|C|+|S|$ with the vertex $n$. Thus, in the graph $\widetilde{G}$, each vertex in $S$ is connected to every vertex in $R$. Then, in $\widetilde{G}, d_{i, j}=1$ for $i \in S$ and $j \in R$. Let $\mathbf{e}_{i}$ the $i$-th standard basis vector in $\mathbb{R}^{n}$. Let $\mathbf{z} \in \mathbb{R}^{n}$ given by

$$
\mathbf{z}=(n-|C|-|S|) \mathbf{e}_{1}-\sum_{j=|C|+|S|+1}^{n} \mathbf{e}_{j}
$$

Since $\operatorname{diam}(G)=2$, an easy computation shows that $\partial+2=2 n-|S|$. We claim that $\mathcal{L}(\widetilde{G}) \mathbf{z}=(\partial+2) \mathbf{z}$. In fact,

$$
\begin{aligned}
& \mathcal{L}(\widetilde{G}) \mathbf{z}=(n-|C|-|S|) \mathcal{L}(\widetilde{G})\left(\mathbf{e}_{1}\right)-\sum_{j=|C|+|S|+1}^{n} \mathcal{L}(\widetilde{G})\left(\mathbf{e}_{j}\right) \\
& =(n-|C|-|S|) \operatorname{Col}_{1}(\mathcal{L}(\widetilde{G}))-\sum_{j=|C|+|S|+1}^{n} \operatorname{Col}_{j}(\mathcal{L}(\widetilde{G})) \\
& =(n-|C|-|S|)\left[\begin{array}{c}
\partial \\
-2 \\
\vdots \\
-2 \\
-1 \\
\vdots \\
-1 \\
-2 \\
-2 \\
\vdots \\
-2
\end{array}\right]-\left[\begin{array}{c}
-2 \\
-2 \\
\vdots \\
-2 \\
-d_{|C|+1,|C|+|S|+1} \\
\vdots \\
-d_{|C|+|S|,|C|+|S|+1} \\
-d_{|C|+|S|+2,|C|+|S|+1} \\
\vdots \\
-d_{n,|C|+|S|+1}
\end{array}\right]-\cdots-\left[\begin{array}{c}
-2 \\
-2 \\
\vdots \\
-2 \\
-d_{|C|+1, n} \\
\vdots \\
-d_{|C|+|S|, n} \\
-d_{|C|+|S|+1, n} \\
-d_{|C|+|S|+2, n} \\
\vdots \\
\operatorname{Tr}\left(v_{n}\right)
\end{array}\right]
\end{aligned}
$$




$$
\begin{aligned}
& =(n-|C|-|S|)\left[\begin{array}{c}
\partial \\
-2 \\
\vdots \\
-2 \\
-1 \\
\vdots \\
-1 \\
-2 \\
-2 \\
\vdots \\
-2
\end{array}\right]-\left[\begin{array}{c}
-2(n-|C|-|S|) \\
-2(n-|C|-|S|) \\
\vdots \\
-2(n-|C|-|S|) \\
-\sum_{j=|C|+|S|+1}^{n} d_{|C|+1, j} \\
\operatorname{Tr}\left(v_{|C|+|S|+1)} \sum_{j=|C|+|S|+1}^{n} d_{|C|+|S|+1, j}\right. \\
\operatorname{Tr}\left(v_{|C|+|S|+2)} d_{j=|C|+|S|+1}^{n} d_{|C|+|S|+2, j}\right. \\
\vdots \\
\operatorname{Tr}\left(v_{n}\right)-\sum_{j=|C|+|S|+1}^{n} d_{n, j}
\end{array}\right] \\
& =\left[\begin{array}{c}
(n-|C|-|S|)(\partial+2) \\
0 \\
\vdots \\
0 \\
0 \\
\vdots \\
0 \\
-(2 n-|S|) \\
-(2 n-|S|) \\
\vdots \\
-(2 n-|S|)
\end{array}\right]
\end{aligned}
$$

The last equality is a consequence of

$$
-\sum_{j=|C|+|S|+1}^{n} d_{i, j}=|C|+|S|-n, \quad \text { for each } i=|C|+1, \ldots,|C|+|S|
$$

and

$$
\operatorname{Tr}\left(v_{i}\right)-\sum_{j=|C|+|S|+1}^{n} d_{i, j}=2|C|+|S|, \quad \text { for each } i=|C|+|S|+1, \ldots, n
$$


Therefore,

$$
\mathcal{L}(\widetilde{G}) \mathbf{z}=\left[\begin{array}{c}
(n-|C|-|S|)(\partial+2) \\
0 \\
\vdots \\
0 \\
0 \\
\vdots \\
0 \\
-(\partial+2) \\
-(\partial+2) \\
\vdots \\
-(\partial+2)
\end{array}\right]=(\partial+2) \mathbf{z} .
$$

Define $D=\mathcal{L}(G)-\mathcal{L}(\widetilde{G})$. The entries of $D$ are zeros except for the entries

$$
D(|C|+|S|,|C|+|S|)=D(n, n)=1 \quad \text { and } \quad D(|C|+|S|, n)=D(n,|C|+|S|)=-1 .
$$

One can easily see that $\mathbf{z}^{T} D \mathbf{z}=1$. Let $\mathbf{z}_{0}=\frac{\mathbf{z}}{\|\mathbf{z}\|_{2}}$. Rayleigh-Ritz Theorem [8] implies that $\partial_{1}^{L}(G) \geq \mathbf{z}_{0}^{T} \mathcal{L}(G) \mathbf{z}_{\mathbf{0}}$ and $\mathbf{z}_{0}^{T} \mathcal{L}(\widetilde{G}) \mathbf{z}_{\mathbf{0}}=\partial+2$. Therefore,

$$
\begin{aligned}
\partial+2=\partial_{1}^{L}(G) \geq \mathbf{z}_{0}^{T} \mathcal{L}(G) \mathbf{z}_{0} & =\mathbf{z}_{0}^{T} \mathcal{L}(\widetilde{G}) \mathbf{z}_{0}+\mathbf{z}_{0}^{T} D \mathbf{z}_{0} \\
& =\partial+2+\mathbf{z}_{0}^{T} D \mathbf{z}_{0}=\partial+2+\frac{1}{\|\mathbf{z}\|_{2}^{2}}>\partial+2,
\end{aligned}
$$

which is a contradiction. Consequently, in the graph $G$ each vertex in $S$ is connected to every vertex in $R . \square$

THEOREM 3.4. Let $G$ be a connected graph on $n$ vertices with a cluster $(C, S)$. Let $\partial$ be the transmission in $G$ of the vertices in $C$. Let $H$ be a graph of order $|C|$. The distance Laplacian spectral radii of $G$ and $G(H)$ are equal in the following cases:

1. $\operatorname{diam}(G) \geq 3$,

2. $\operatorname{diam}(G)=2$ and $\partial$ is not the largest transmission in $G$, and

3. $\operatorname{diam}(G)=2, \partial$ is the largest transmission in $G$ and $|C|+|S|<n$.

Proof. As in the proof of Theorem 3.2, because of Theorem 2.6, it suffices to show the results for $H=K_{|C|}$. Since $C$ is a set of independent vertices, $G \neq K_{n}$. Let $D$ be the largest transmission in $G$.

1. Assume $\operatorname{diam}(G) \geq 3$. From Theorem 2.5, $\partial_{1}^{L}(G)>D+2$. Then $\partial_{1}^{L}(G)>\partial+2$. Therefore, from Theorem 3.1, $\partial_{1}^{L}(G)$ is an eigenvalue of the matrix $T$ defined in (3.1). The eigenvalues of $T$ are also eigenvalues of $\mathcal{L}\left(G\left(K_{|C|}\right)\right)$. Finally, since $\partial_{1}^{L}(G) \geq \partial_{1}^{L}\left(G\left(K_{|C|}\right)\right)$, these distance Laplacian spectral radii are equal.

2. Assume that $\operatorname{diam}(G)=2$ and $\partial$ is not the largest transmission in $G$. From Theorem $2.5, \partial_{1}^{L}(G) \geq$ $D+2>\partial+2$. The rest of the proof runs as in 1.

3. Assume now that $\operatorname{diam}(G)=2, \partial$ is the largest transmission in $G$ and $|C|+|S|<n$. We have $\partial_{1}^{L}(G)>\partial+2$ or $\partial_{1}^{L}(G)=\partial+2$. As above, the result follows if $\partial_{1}^{L}(G)>\partial+2$. Suppose that $\partial_{1}^{L}(G)=\partial+2$. We apply Lemma 3.3, to get that each vertex in $S$ is connected to every vertex in 
$R$. We can proceed as in the proof of Lemma 3.3, to obtain that $\mathcal{L}(G) \mathbf{z}=\partial_{1}^{L}(G) \mathbf{z}$ with $\mathbf{z}$ as in (3.3). We claim that $\partial_{1}^{L}(G)$ has multiplicity at least $|C|$. Consider the following set of vectors in $\mathbb{R}^{n}$

$$
\mathcal{F}=\left\{\mathbf{x}_{1}, \mathbf{x}_{2}, \ldots, \mathbf{x}_{|C|-1}, \mathbf{z}\right\},
$$

where

$$
\mathbf{x}_{i}=\mathbf{e}_{1}-\mathbf{e}_{i+1}, \quad i=1, \ldots,|C|-1 .
$$

Clearly, $\mathcal{F}$ is a linearly independent set. To prove that $\partial_{1}^{L}(G)$ has multiplicity at least $|C|$, it remains to show that each vector $\mathbf{x}_{i}$ in $\mathcal{F}$ is an eigenvector of $\mathcal{L}(G)$ corresponding to $\partial_{1}^{L}(G)$. For $i=1, \ldots,|C|-1$, we may write $\mathbf{x}_{i}=\left(\widetilde{\mathbf{x}}_{i}, \mathbf{0}\right)$, where $\widetilde{\mathbf{x}}_{i}=\mathbf{e}_{1}-\mathbf{e}_{i+1} \in \mathbb{R}^{|C|}$ and $\mathbf{0} \in \mathbb{R}^{n-|C|}$. For $i=1, \ldots,|C|-1$, we have $E \widetilde{\mathbf{x}}_{i}=(\partial+2) \widetilde{\mathbf{x}}_{i}$, where $E$ is as in (3). From (3), we get that $\mathbf{x}_{i}$, $i=1, \ldots,|C|-1$, is an eigenvector of $\mathcal{L}(G)$ corresponding to $\partial_{1}^{L}(G)$. Thus, we have proved that each vector in the linearly independent set $\mathcal{F}$ is an eigenvector of $\mathcal{L}(G)$ for the eigenvalue $\partial_{1}^{L}(G)$. Therefore, $\partial_{1}^{L}(G)$ has multiplicity at least $|C|$. We apply Theorem 3.1 to the graph $G$, to obtain that $\partial_{1}^{L}(G)$ is an eigenvalue of $T$, and thus, $\partial_{1}^{L}(G)$ is also an eigenvalue of $\mathcal{L}\left(G\left(K_{|C|}\right)\right)$.

The proof is complete.

Let $k \geq 2$. Let $G\left(H_{i}: i \in N_{k}\right)$ as in Definition 1.4. For $i=1, \ldots, k$, let $\partial_{i}$ be the common transmission in $G$ of the vertices in $C_{i}$. Since $k \geq 2$ and the clusters are pairwise disjoint, it follows that $\operatorname{diam}(G) \geq 3$. Observe that, for $i=1, \ldots, k$,

$$
G\left(H_{j}: j \in N_{k}\right)=G\left(H_{j}: j \in N_{k} \backslash\{i\}\right)\left(H_{i}\right) .
$$

Repeated application of the above results enables us to obtain

TheOREM 3.5. Let $G$ be a connected graph having pairwise disjoint clusters $\left(C_{1}, S_{1}\right), \ldots,\left(C_{k}, S_{k}\right)$. For $i=1, \ldots, k$, we have

1. $\partial_{i}+2$ is an eigenvalue of $\mathcal{L}(G)$ with multiplicity at least $\left|C_{i}\right|-1$,

2. $\partial_{i}-\left|C_{i}\right|+2$ is an eigenvalue of $\mathcal{L}\left(G\left(K_{\left|C_{j}\right|}: j \in N_{k}\right)\right)$ with multiplicity at least $\left|C_{i}\right|-1$,

3. the matrices $\mathcal{L}(G)$ and $\mathcal{L}\left(G\left(H_{j}: j \in N_{k}\right)\right)$ have $n+\sum_{j=1}^{k}\left|C_{j}\right|-k$ eigenvalues in common, and

4. the distance Laplacian spectral radii of $\mathcal{L}(G)$ and $\mathcal{L}\left(G\left(H_{j}: j \in N_{k}\right)\right)$ are equal.

Acknowledgements. The authors are very grateful to an anonymous referee for all his/her comments and corrections.

\section{REFERENCES}

[1] N.M.M. Abreu, D.M. Cardoso, E.A. Martins, M. Robbiano, and B. San Martín. On the Laplacian and signless Laplacian spectrum of a graph with k pairwise co-neighbor vertices. Linear Algebra Appl., 437:2308-2316, 2012.

[2] M. Aouchiche and P. Hansen. Two Laplacians for the distance matrix of a graph. Linear Algebra Appl., 439:21-33, 2013.

[3] M. Aouchiche and P. Hansen. Some properties of the distance Laplacian eigenvalues of a graph. Czechoslovak Math. J., 64(3):751-761, 2014.

[4] D.M. Cardoso and O. Rojo. Edge perturbation on graphs with clusters: Adjacency, Laplacian and signless Laplacian eigenvalues. Linear Algebra Appl., 512:113-128, 2017. 
[5] P.J. Davis. Circulant Matrices. Wiley, New York, 1979.

[6] J.-M. Guo. The effect on the Laplacian spectral radius of a graph by adding or grafting edges. Linear Algebra Appl., 413:59-71, 2006.

[7] M. Fiedler. Algebraic connectivity of graphs. Czechoslovak Math. J., 23:298-305, 1973.

[8] R. Horn and C.R. Johnson. Matrix Analysis. Cambridge University Press, Cambridge, 1985.

[9] R. Merris. Laplacian matrices of graphs: A survey. Linear Algebra Appl., 197/198:143-176, 1994.

[10] G. Pastén and O. Rojo. Laplacian spectrum, Laplacian-energy-like invariant, and Kirchhoff index of graphs constructed by adding edges on pendent vertices. MATCH Commun. Math. Comput. Chem., 73:27-40, 2015.

[11] O. Rojo. Effects on the energy and Estrada indices by adding edges among pendent vertices. MATCH Commun. Math. Comput. Chem., 74:343-358, 2015.

[12] J.Y. Shao, J.M. Guo, and H.Y. Shan. The ordering of trees and connected graphs by algebraic connectivity, Linear Algebra Appl., 428:1421-1438, 2008.

[13] J. Xue, H. Lin, K.Ch. Das, and J. Shu. More results on the distance (signless) Laplacian eigenvalues of graphs. Preprint, arXiv:1705.07419v1, 2017. 